Accepted for publication in J.Appl.Phys., Vol. 93, No. 12, June 15, 2003.

\title{
Bias and Self-Bias of Magnetic Macroparticle Filters for Cathodic Arc Plasmas
}

\author{
Eungsun Byon and André Anders
}

\author{
Building Technologies Department \\ Environmental Energy Technologies Division \\ Ernest Orlando Lawrence Berkeley National Laboratory \\ University of California \\ 1 Cyclotron Road \\ Berkeley, California 94720-8223
}

December 2002

This work was supported by the Assistant Secretary for Energy Efficiency and Renewable Energy, Office of Building Technology, of the U.S. Department of Energy under Contract No. DE-AC03-76SF00098. E. Byon was supported by the post-doctoral fellowship program of the Korean Science and Engineering Foundation (KOSEF). 


\title{
Bias and Self-Bias of Magnetic Macroparticle Filters for Cathodic Arc Plasmas
}

\author{
Eungsun Byon a and André Anders ${ }^{\mathrm{b}}$ \\ Building Technologies Department, Environmental Energy Technologies Division \\ Lawrence Berkeley National Laboratory, University of California
}

\begin{abstract}
Curved magnetic filters are often used for the removal of macroparticles from cathodic arc plasmas. This study addresses the need to further reduce losses and improving plasma throughput. The central figure of merit is the system coefficient, $\kappa$, defined as filtered ion current normalized by the plasma-producing arc current. The coefficient $\kappa$ is investigated as a function of continuous and pulsed magnetic field operation, magnetic field strength, external electric bias, and arc amplitude. It increases with positive filter bias but saturates at about $15 \mathrm{~V}$ for relatively low magnetic field $(\sim 10 \mathrm{mT})$, whereas stronger magnetic fields lead to higher $\kappa$ with saturation at about $25 \mathrm{~V}$. Further increase of positive bias reduces $\kappa$. These findings are true for both pulsed and continuous filters. Bias of pulsed filters has been realized using the voltage drop across a self-bias resistor, eliminating the need for a separate bias circuit. Almost $100 \mathrm{~A}$ of filtered copper ions have been obtained in pulsed mode, corresponding to $\kappa \approx 0.04$. The results are interpreted by a simplified potential trough model.
\end{abstract}

\section{INTRODUCTION}

Cathodic arc plasmas ${ }^{1}$ are widely used in industrial coatings of hard, protective, and decorative films on a variety of substrates, including cutting tools, automotive components, and building appliances such as door knobs and water faucets. In most of these applications, the arc discharge operates in a reactive gas environment, forming compound films such as TiN, TiAlN, $\mathrm{ZrN}, \mathrm{NbN}, \mathrm{TiO}_{2}$, and $\mathrm{Al}_{2} \mathrm{O}_{3}$. Macroparticle contamination is an issue but has been tolerated in these applications. Macroparticle production ${ }^{2}$ occurs at cathode spot and is intimately related to the explosive plasma formation at arc cathode spots. ${ }^{3,4}$

Demand for films of higher quality and expansion of this coating technology into semiconductor ${ }^{5,6}$ and optical ${ }^{7}$ markets requires development and implementation of next generation processes and equipment. Various approaches have been developed to rid cathodic arc plasma from macroparticles, ${ }^{8-11}$ and magnetic filtering is to date the most promising and successful.

Plasma filtering was first developed in the 1970s, Ref. ${ }^{12,13}$, and the principle has not much changed since. The plasma is guided to a substrate which is not in line-of-sight from the cathode, thereby plasma particles (ions, electrons) are separated from the much heavier macroparticles that move along almost straight paths due to their inertia. The electrons of the streaming cathodic arc plasma are magnetized, i.e. they gyrate many times around a magnetic field line before they suffer a collision. In a collision, an electron's guiding center (the center of gyration drifting along the field line) is displaced to a magnetic field line which is about one gyration radius away from the original field line. The magnetic field of a filter is usually not sufficiently strong to also magnetize ions. The motion of ions is therefore not strongly (directly) affected by the magnetic field. The motion of ions is determined by the electric field that can exist in magnetized plasma. Each magnetic field line can be considered as an equipotential line, the electric potential being communicated along the line via electrons. Perpendicular to the magnetic field lines, ions have a higher mobility than electrons, as will be illustrated later. As a consequence, ions can leave the central region of the plasma, and the plasma flow appears "polarized" with a negative potential along the central axis and a positive potential near the filter wall. ${ }^{14}$ As a result of the combined action of magnetic field (mainly on electrons)

\footnotetext{
${ }^{a}$ Permanent address: Surface Engineering Department, Korean Institute of Machinery and Materials, 16 SangnamDong, Changwon, 641-010, Korea.

${ }^{\mathrm{b}}$ Author to whom correspondence should be submitted: E-mail aanders@lbl.gov
} 
and electric field (mainly on ions), and taking electron-ion coupling into account, the plasma stream as a whole through the filter.

As already shown in the pioneering work of Aksenov and coworkers, ${ }^{12,13,15}$ biasing the filter positively improves plasma throughput and thus deposition rate. They have also shown that a floating wall may assume a positive potential with respect to the plasma due to the greatly reduced mobility of electrons perpendicular to the magnetic field lines. A filter may be positively biased by external means to enhance ion throughput, but also a floating filter can assume positive potential if the magnetic field is sufficiently strong and essentially parallel to the filter wall. ${ }^{16}$

Plasma losses in a filter are maximum at the outside curvature of a filter because plasma has a tendency to move there due to inertia. Bilek and coworkers ${ }^{17}$ utilized this feature and introduced a biased strip located along the wall near the outside curvature. They have shown that such biased strip electrode has approximately the same effect than biasing the whole filter body, which was very recently confirmed by Zhang et al. ${ }^{18}$ The plasma, however, also drifts perpendicular to the magnetic field vector and the vector pointing outward from the center of filter curvature, ${ }^{19,20}$ which is also known as off-plane shift. ${ }^{20,21}$ Such shift can be taken into account or even utilized for out-of-plane filters such as the Singapore double-bent design ${ }^{22}$ or the Twist-Filter design ${ }^{23}$.

There are several models for plasma transport in magnetic filters. ${ }^{18,24-26}$ Modeling is not simple because the problem

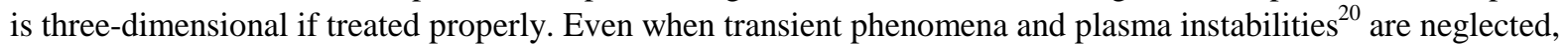
simplifying assumptions have to be made to obtain useful steady-state results. Despite all the progress in understanding and implementation of curved magnetic filters, there is still a need for further work that addresses questions of possible limits of plasma transport (such as recently indicated by You et al. ${ }^{26}$ ), transport as a function of ion mass, plasma density, pulsed versus DC operation, filter size, and open versus closed filter geometry, just to name a few of the important parameters and issues. The present study aims to explore part of this wide parameter space, thereby contributing to ultimately formulate filter theory and to develop practical design criteria for minimum plasma losses in filters.

\section{SYSTEM COEFFICIENT}

In order to compare results of different configurations or parameter settings, it is useful to define the system coefficient as

$$
\kappa=\frac{I_{\text {ion }}}{I_{\text {arc }}},
$$

which is the useable (filtered) ion current normalized by the arc current. This is useful because the arc current is known to be proportional to the amount of plasma produced at cathode spots. The advantage of using $\kappa$ as opposed to filter efficiency defined as (ion output current / ion input current) is twofold. First, there is no need to determine the ion current entering the filter, a quantity usually not known or difficult to measure. Second, the coefficient $\kappa$ describes the overall system performance, including source efficiency and source-filter coupling. Note that $\kappa$ is not a conventional efficiency because, unlike conventionally defined efficiencies, $\kappa$ can never reach $100 \%$ since most of the arc current is transported by electrons. Looking at reports in the literature, typical system coefficients for filtered arcs are about $1 \%$, and values of $2-4 \%$ can be considered high.

For pulsed systems, the definition (1) of the system coefficient may be questionable if current pulses do not have a nice flat portion. In these cases, it is more relevant to consider the charge transferred to the substrate per pulse, normalized by the charge of the arc pulse eroding the cathode, i.e.,

$$
\kappa_{p}=\frac{\int I_{\text {ion }}(t) d t}{\int I_{\text {arc }}(t) d t},
$$

where the integral is to be taken over the duration of a pulse. The integration is easy to accomplish with digital data acquisition. Definition (2) becomes (1) for continuous operation.

In the following study, the performance of a $90^{\circ}$-curved filter was investigated in magnetic pulsed and DC mode, with the goal to maximize the system coefficient. 


\section{EXPERIMENTAL}

A cathodic arc plasma source of the "minigun" type ${ }^{27}$ was used, which essentially consisted of a cathode rod of 6.25 $\mathrm{mm}$ diameter mounted on axis of a grounded, annular anode cylinder of $2.5 \mathrm{~cm}$ inner diameter. The anode opening, where the plasma streams from the source, was $20 \mathrm{~mm}$ from the cathode plane (Figure 1). Cathode spots can burn on the front face of the cathode rod, eroding the material and forming fully ionized metal plasma. Copper was used as the cathode material. The cathode rod was hold in an alumina ceramic tube.

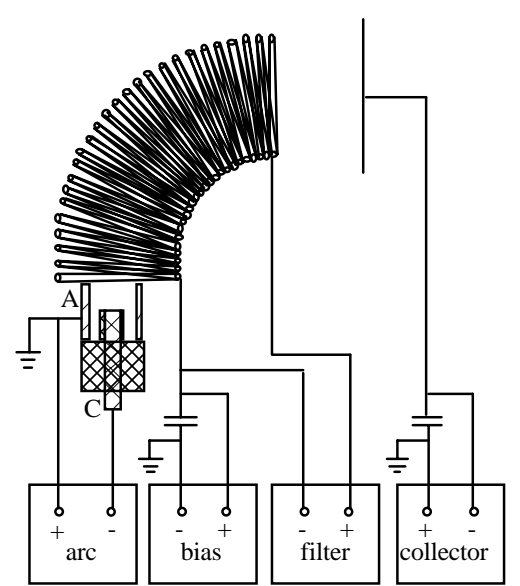

Figure 1. Experimental setup for biased open-coil filter operating in DC mode.
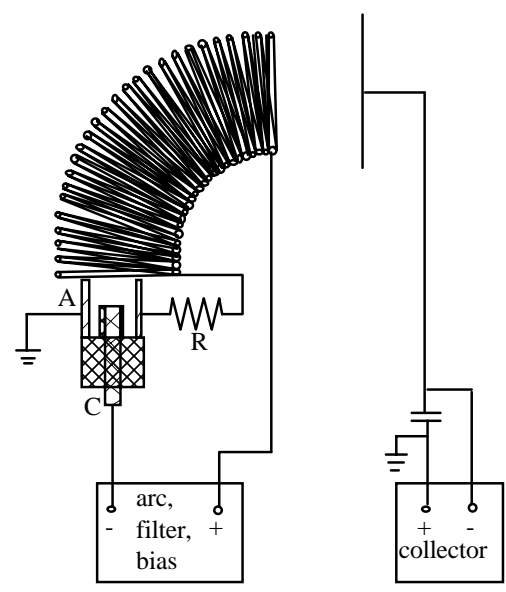

Figure 2. Experimental setup for biased open-coil filter operating in pulsed mode.
The plasma source was operated in pulsed mode fed by either a 10-stage 1$\Omega$ pulse forming network ${ }^{28}$ (PFN) for currents exceeding 200 A, or by a transistor-switched DC supply for arc currents less than $200 \mathrm{~A}$. Each arc current pulse has a rise and fall time of about $50 \mu \mathrm{s}$ and $150 \mu \mathrm{s}$, respectively, and a central flat portion of about $400 \mu$ s. This steady-state portion can be used to evaluate the steady-state performance of a filter system even when operated with pulsed arc plasma. The arc pulse repetition rate was about 1 pulse per second. The amplitude of the arc current depended on the charging voltage of the PFN, or on the open-circuit setting if the DC supply was used. The anode of the plasma source was grounded. The vacuum chamber was cryogenically pumped with a base pressure of about $5 \times 10^{-5} \mathrm{~Pa}$.

The filter consisted of a quarter-torus solenoid formed by a bent coil of 26 turns of water-cooled copper tubing of $6.25 \mathrm{~mm}$ outer diameter. The major and minor radius of the torus were $R_{t}=150 \mathrm{~mm}$ and $r_{t}=36 \mathrm{~mm}$, respectively. The plasma source was placed such as to inject a plasma stream into the filter (Figures. 1 and 2). The magnetic field was measured with DC operation using a commercial Hall probe. In the center of the filter, the axial component of the magnetic field increased linearly with filter coil current at a rate of $74 \mu \mathrm{T} / \mathrm{A}$. For example, if the coil is operated at $500 \mathrm{~A}$, the field is $37 \mathrm{mT}$, which is quite typical for magnetic filters.

The filter coil could be operated in two modes: (i) pulsed mode, usually electrically in series with the arc current, or (ii) DC mode, powered by an electrically floating, high-current power supply. Water cooling of the coil was accomplished using insulated high-current feedthroughs. An external electric bias potential could be applied to the filter, or the filter could intentionally be grounded or floated. Figure 1 shows the application of filter bias for the DC mode. The bias capacitor serves as a charge "reservoir," ensuring that the bias potential stays constant despite the neepd for a large bias current during the arc pulse. With the application of the bias capacitor, and assuming pulsed operation of the plasma source, the bias power supply could be relatively small, e.g. with current of only 1-10 A for voltages up to $50 \mathrm{~V}$.

For systems operating with pulsed arcs and "open" filters ${ }^{8}$, it is practical and economical to use the arc current for the generation of the magnetic filter field. This approach eliminates the need for a magnet power supply and also reduces the overall power consumption and cooling needs since the filter coil is only used for the duty cycle

$$
\delta=\frac{t_{\text {pulse }}}{t_{\text {pulse }}+t_{\text {off }}},
$$

which is typically a few percent. This solution has the additional advantage that higher currents can be utilized than one would use for DC systems, and higher currents can be advantageous for obtaining highest system coefficients. For example, system coefficients up to $3.5 \%$ were demonstrated with the pulsed Twist Filter ${ }^{23}$ for carbon. A 
disadvantage of pulsed operation is that despite higher arc and filter current, the overall time-averaged deposition rates are usually less than the rates obtained with DC arcs and DC filters. Additionally, high-current pulsed systems must be built to accommodate the repetitive mechanical stress imposed by the pulsed current and its pulsed magnetic field.

Up to now, the series connection of arc and filter was usually done directly. Here, to our knowledge for the first time, we discuss biasing a pulsed filter of the open-coil style. A different technique of biasing needs to be applied when arc source and filter are operated in pulsed mode fed by a pulse-forming-network (PFN). This is due to the fact that the bias capacitor couples to the PFN, i.e., essentially becoming part of it. An elegant solution is using a lowohm resistor introduced between the anode of the arc source and the filter entrance (Figure 2). The "self-bias" obtained follows simply from Ohm's law

$$
V_{S B}=R_{S B} I_{a r c}^{*},
$$

where $R_{S B}$ is the "self-bias" resistor, and $I_{\text {arc }}^{*}$ is the fraction of the arc current that flows through the plasma source anode and the self-bias resistor. The star indicates that not all of the arc current flows this way, $I_{a r c}^{*}<I_{a r c}$, since some electrons emitted by the cathode will drift to the turns of the filter coil, bypassing the designated source anode. The bias resistor should be selected in the milliohm range. For example, a current of $300 \mathrm{~A}$ through a $50 \mathrm{~m} \Omega$ resistor will cause a voltage drop of $15 \mathrm{~V}$, which is known to be about the right amount needed to improve transport of plasma. High-precision (1\%) resistors were directly mounted between the source anode and the filter coil. Resistors of nominal $50 \mathrm{~W}$ power were used (power rating for continuous operation). The metal case of the resistor used was mounted on the water-cooled anode of the plasma source to achieve stability of resistance and to avoid overheating.

The system coefficient was determined using a negatively biased ion collector as shown in Figures. 1 and 2. It is important (i) to use a collector capacitor that ensures constant collector bias throughout each pulse, (ii) to make sure that "arcing" on the collector, if it occurs, is detected and data are removed from the data collection, (iii) to use a negative collector bias large enough to ensure ion saturation, and (iv) to use a negative collector bias small enough to permit neglecting of secondary electron emission from the ion collector. Before taking lots of data, we determined that a negative collector bias of $90 \mathrm{~V}$ was suitable. The collector capacitor had a capacity of $1500 \mu \mathrm{F}$. Current measurements were done via wide-band Pearson current transformers (model 1114 with 0.01 V/A, and model 110 with $0.1 \mathrm{~V} / \mathrm{A}$ ). Data acquisition was accomplished using a $500 \mathrm{MHz}, 2$ Gsample/s, 4-channel digital oscilloscope (TDS 744).

Each data point in all measurements represents the average of 50 individual measurements. Averaging is needed due to the "noisy," explosive character of arc plasma formation, which makes individual measurements "scatter." This scattering is not related to inadequate measuring devices but represents real fluctuation of physical quantities such as plasma density, ion velocity, and charge states. Only by averaging we can present insightful trends. One should always bear in mind that all data are average and that momentary, local values do fluctuate around average values.

In the Introduction it was mentioned that ions can have a higher mobility perpendicular to the magnetic field lines than electrons, which can lead to a positive floating potential with respect to the plasma potential. Measurements of the floating potential of the filter have been carried out using a 10:1 high-impedance voltage probe connected to the exit turn of the filter coil.

An approximate radial potential distribution in the exit plane of the filter was determined using a small floating probe that was carefully positioned across the filter diameter using a movable feedthrough. The floating probe was a small cylindrical wire extending $2 \mathrm{~mm}$ beyond its ceramic housing. The probe wire was connected to a highimpedance input connector of the oscilloscope. The reference potential for these measurements was ground. Although the floating potential of the probe must not be confused with the local plasma potential, the shape of the potential curve is a very good indication for the physical situation since one can assume that the difference between plasma potential and floating potential does not vary strongly when considering different locations, hence $\partial V_{\text {plasma }} / \partial R \approx \partial V_{\text {float }} / \partial R$. The derivative, not the absolute potential, is of physical importance since it represents the electric field acting on charged particles, as further discussed in section V. 
Source-filter coupling is known to be important for the system performance, and it is one of the reasons why characterization by the system coefficient was chosen rather than focusing on filter efficiency. Coupling includes geometric details such as size and position of the plasma source anode as well as the magnetic field distribution. Obviously, geometry and field alone offer a wide range of variability. For the sake of transparency of this study, we kept the geometry constant, with the exit plane of the source anode coinciding with the entrance plane of the filter.

\section{RESULTS}

\section{Filter With Continuous Magnetic Field}

Both magnetic field strength and filter bias are known to have an effect on the efficiency of plasma transport through the filter. Using an arc current of $100 \mathrm{~A}$, one can see from Figure 3 that the system coefficient is greatly improved with increasing positive bias at otherwise constant conditions.

Figure 4 shows the effect of increasing bias, at a fixed filter coil current of $500 \mathrm{~A}$, as a function of arc current. As expected, the ion current increases with bias (better transport in filter) and with arc current (more plasma produced). These results will be discussed in section $\mathrm{V}$.

\section{Filter With Pulsed Magnetic Field}

Figure 5 shows the effect of the self-bias resistor, which can be quite dramatic. The shift in filter potential caused by the self-bias resistor may improve or reduce plasma transport, depending on the magnitude of the bias obtained. For the specific conditions of our experiment, increasing the self-bias resistor up to $25 \mathrm{~m} \Omega$ improved the system coefficient significantly, but higher resistor values caused bias shifts that are too high, which of course was more pronounced at higher currents. To show the bias shift by the resistors explicitly, the potential at the entrance of the filter was measured using a Tektronix voltage probe (Figure 6). Indeed, self-bias resistor values greater than $25 \mathrm{~m} \Omega$ cause bias voltages at high currents exceeding $25 \mathrm{~V}$, i.e. values which DC evaluation qualified as excessive.

\section{Potential Measurements}

Figure 7 shows the floating potential of a filter as a function of the filter coil current, i.e. as a function of its magnetic field. As expected, the "wall" of the filter (the bare turns of the copper coil) became more positive at stronger magnetic field, which enhanced the wall height of the potential valley or "trough" in the filter.

The potential trough was also measured using a small movable floating probe. Figure $\mathbf{8}$ shows the potential of a floating probe at different positions in the filter exit plane determined for three different external filter bias voltages.

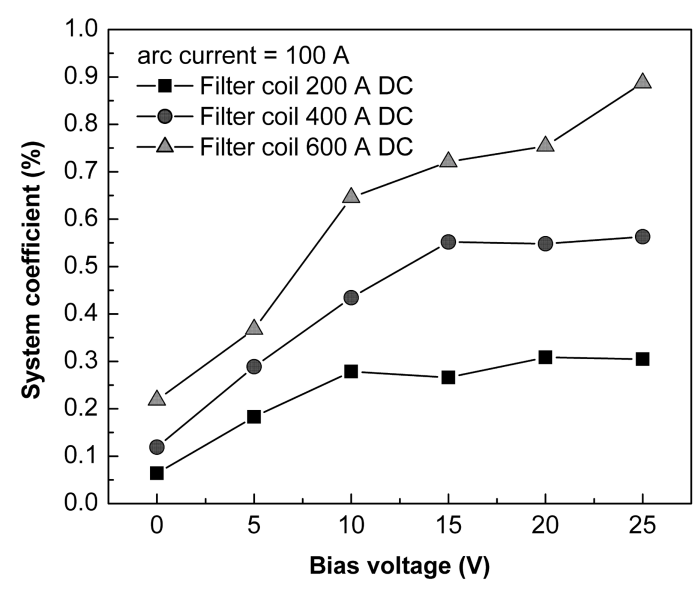

Figure 3. System coefficient as a function of DC filter bias, with the magnetic field (filter coil current) as a parameter; arc current $100 \mathrm{~A}$.

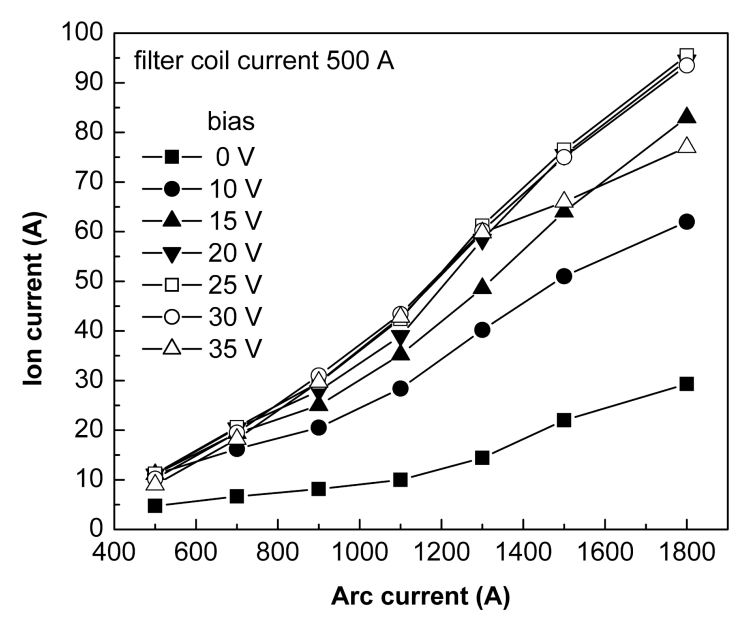

Figure 4. Filtered ion current as a function of arc current with filter bias as a parameter. The magnetic field was constant, generated by a coil current of $500 \mathrm{~A}$. 


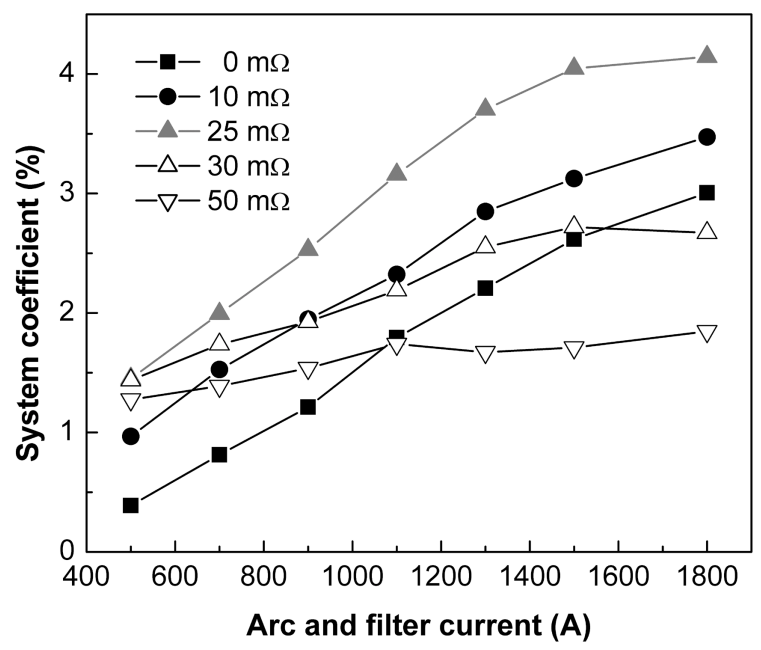

Figure 5. Effect of the self-bias resistor on the filtered ion current. Nominally zero $\Omega$ correspond to direct series connection between plasma source

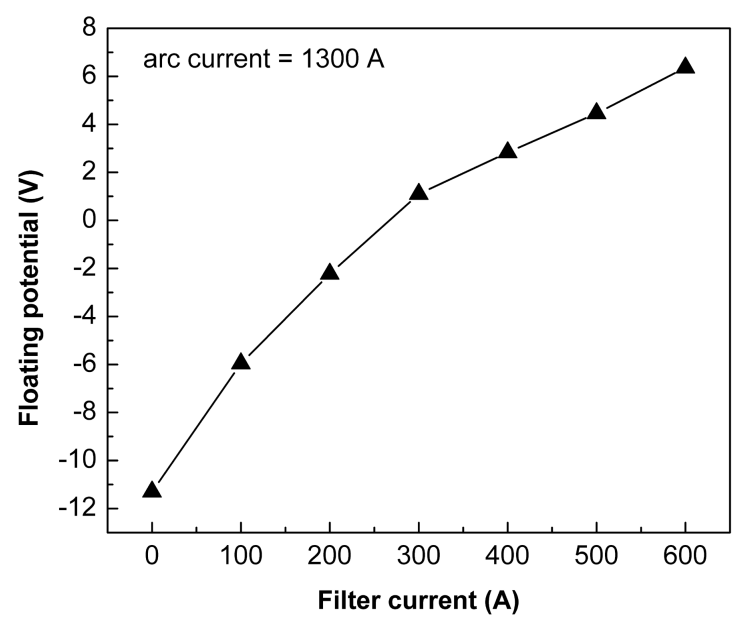

Figure 7. Floating potential of the filter coil as a function of current flowing through the coil, i.e. as determined by the magnetic field inside the filter.

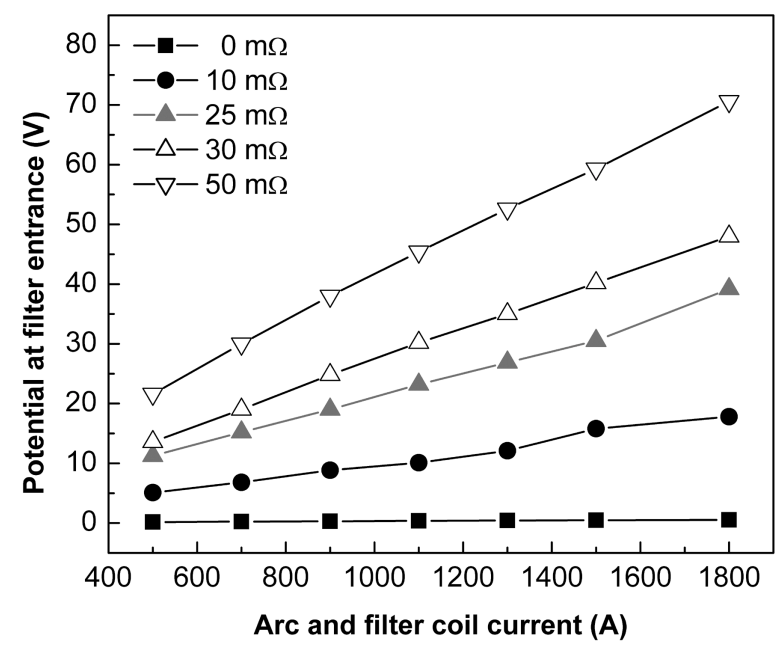

Figure 6. Electric potential at the filter entrance as a function of arc and filter current (in series) with the self-bias resistor as a parameter.

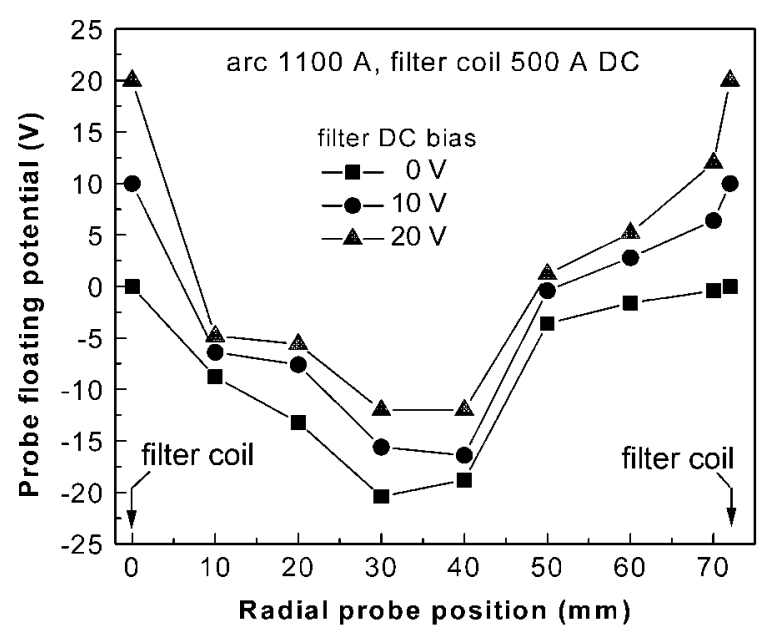

Figure 8. Potential of a floating probe at different positions in the filter exit plane determined at three different filter bias. Zero and $72 \mathrm{~mm}$ in radial position corresponds to the inner diameter of the filter coil, with zero at smaller filter curvature (the "inner" wall of the curved filter). Potentials shown at zero and $72 \mathrm{~mm}$ are filter bias, not the probe's.

\section{DISCUSSION}

\section{Filter With Continuous Magnetic Field}

Confirming previous results, Figure 3 shows that positive bias improves the system coefficient. Figure 3 indicates that in order to fully utilize higher bias, the magnetic field of the filter needs to be stronger. At relatively weak magnetic field $(\sim 10 \mathrm{mT})$, the system coefficient seems to saturate while at stronger fields, more plasma can be transported at higher bias. 
From Figure 4 one can see that even as little as $10 \mathrm{~V}$ of bias improved the transport of plasma significantly. Further increase of bias was beneficial up to about $25 \mathrm{~V}$, whereas higher bias lead to reduction. These measurements confirmed previous findings that bias of about $20 \mathrm{~V}$ is optimum - at last for the typical magnetic fields used (here $B$ $=37 \mathrm{mT}$ at $500 \mathrm{~A}$ coil current). One should note the very high absolute filtered copper ion currents than have been obtained - almost $100 \mathrm{~A}$ for the strongest magnetic field and $20 \mathrm{~V}$ bias.

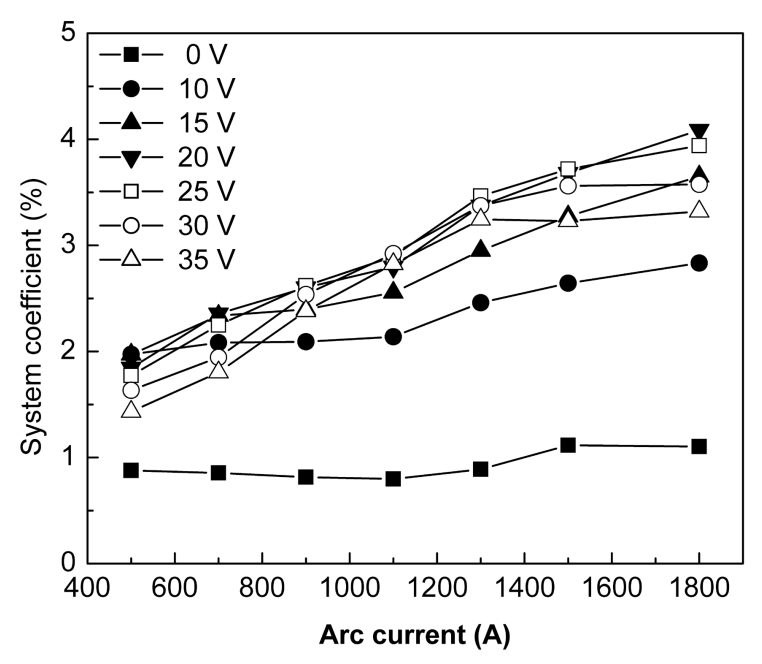

Figure 9. Measurements of Figure 4 displayed in a normalized way using the system coefficient.
In order to identify and evaluate possible limits of the amount of plasma that a given filter can transport, the data of Figure 4 are plotted in Figure 9 using the system coefficient definition (2). The slightly increasing curves imply that the system coefficient does not strongly depend on the amount of plasma being transported, and it appears that the transport is even better for large arc currents. This unexpected result differs from recent observations ${ }^{26}$ made with the off-plane double bend (OFDB) filter where the ion saturation current tended to reach a maximum at an DC arc current of about 120 A. For comparison, the maximum system coefficient was about $1.4 \%$ and $1.7 \%$ for OFDB-systems of $10 \mathrm{~cm}$ and $15 \mathrm{~cm}$ inner diameter. ${ }^{26}$

Based on the results by Storer and coworkers ${ }^{29}$, who found exponential drop of plasma density along the plasma flow path in filters, it was expected that the more compact filter with less curvature investigated here exhibited a higher system coefficient. At a first glance, one could interpret the increase of system coefficient with increasing arc current as improvement of transport, however, taking into account literature data on ion charge states ${ }^{30}$, one realizes that at least part of the questionable effect is due to enhanced ion charge states at increasing arc current and associated magnetic field.

\section{Filter With Pulsed Magnetic Field}

Based on the general knowledge that an optimum positive bias of about $20 \mathrm{~V}$ maximizes the system coefficient, ${ }^{12,16,17}$ it was expected that improvement could also be achieved when the bias was obtained by the voltage drop across an Ohmic resistor. Figure 5 fully confirmed that. With resistance values of a few $\mathrm{m} \Omega$, consistent shifts of the system coefficient to higher values were obtained. For greater resistance values, the bias exceeded the optimum. Since the bias voltage is directly proportional to the current through the resistor, deterioration of the beneficial effect is seen first at high currents. For practical application of the self-bias resistor concept, the arc current must be selected first, and the optimum self-bias resistor value follows. For the specific conditions of our experiment, a self-bias resistor up to $25 \mathrm{~m} \Omega$ improved the system coefficient significantly.

Figure 6, besides giving absolute values of self-bias caused by the resistor, indicates through the linearity of the curves that the current $I_{\text {arc }}^{*}$ flowing through the resistor does not noticeably depend on the absolute value of the arc current. Additionally, one can derive from these data that, for the given geometry, about $80 \%$ of the arc current go to the designated anode of the plasma source.

\section{Floating Potential and Potential Trough}

The results of Figure 7 indicate that the potential trough has a double structure, namely a dip of about $10 \mathrm{~V}$ at about the center of the filter, and a steep potential gradient near the physical wall of the filter (the turns of the coil). While the central section is essentially only shifted when bias voltage is applied to the wall, the outer potential gradient becomes steeper and the trough deeper. That indicates that biasing the filter improves transport of ions that are not confined and guided by the central dip. Photographs of the plasma in open filter coils show that the plasma is moreor-less confined and guided in the center of the filter, depending on the magnetic field strength and coupling of source and filter. Strong magnetic fields lead to strong concentration of the plasma flow along the curved axis of the filter (e.g. Figure 2 of Ref. ${ }^{31}$ ). These findings are corroborated by deposition probe experiments (e.g., see Figure 3 
of Ref..$^{23}$ or Figure 5 of Ref. ${ }^{14}$ ) which show that most plasma streams in a narrow channel whose diameter is much smaller than the inner diameter of the filter.

\section{A Simplified Interpretation of Ion Transport in Curved Filters}

As mentioned above, there have been numerous studies explaining plasma transport in magnetic filters, including the rigid-rotor model introduced by Morozov ${ }^{32}$ in the 1960 s (i.e., even before such structure was used for macroparticle filtering), a similar flux-tube model by Boercker and coworkers ${ }^{33}$, and other hydrodynamic ${ }^{24,26}$ and diffusion ${ }^{18}$ models. Without diminishing the value of these models, one may state that it is not obvious from any of them how an electric potential trough whose "wall height" is typically only 10-20 V is able to guide ions having kinetic energies of 20-150 eV. The wall height of the trough has been measured, for example, by Bilek and coworkers, ${ }^{14}$ and similar data were shown here in Figure 8. The high kinetic ion energies have been determined by many researchers. ${ }^{34-36}$

An answer can be derived from the following simplified picture. Suppose ions are generated at the plasma source with a kinetic energy $E_{k i n}=m_{i} v_{i}^{2} / 2$; this energy refers to a drift motion along an essentially straight path from the source to the filter entrance. After entering the filter, the ions find themselves in a potential channel, or trough, which is the result of electron magnetization and lack magnetization of ions, as confirmed by the existence of a positive floating potential (Figure 7) and probe measurements (Figure 8). The potential trough follows the filter geometry, i.e. it is curved with about the major radius of the torus, $R_{t}$. Due to inertia, the ions will gradually move up the outside wall of the trough and become subject to the force caused by the radial electric field, which accelerate them back in the direction of the trough's minimum, i.e.,

$$
\mathbf{F}_{E}=Q e \mathbf{E}=-Q e \frac{\partial}{\partial R} V(R),
$$

where $Q$ is the ion charge state number and $e$ is the elementary charge. It is known that ions are indeed transported through a curved filter, and therefore the radial electric force must have turned the straight motion in a motion with a curved path (laboratory frame). The force caused by the electric field must have compensated the centrifugal force

$$
\mathbf{F}_{E}=-\mathbf{F}_{R}=\frac{m_{i} v_{i}^{2}}{R^{2}} \mathbf{R}
$$

Considering the radial component one obtains from Eqs.(5) and (6)

$$
\frac{m_{i}}{2} v_{i}^{2}=\frac{1}{2} Q e \frac{\partial V}{\partial R} R
$$

The left-hand side is the kinetic energy which is known to be of order $100 \mathrm{eV}\left(57 \mathrm{eV}\right.$ for copper $\left.{ }^{36}\right)$. The right-hand side tells us that transport equilibrium through the curved filter depends on the radial electric field, $\partial V / \partial R$, the charge state of the ion being transported, $Q$, and the radius of path curvature, $R$, which is approximately given by the major radius of the filter, $R_{t}$. The combination of these parameters according to the right hand side of (7) must be of order $100 \mathrm{eV}$.

To illustrate this consideration one could pick a reasonable example. Suppose the potential trough can be described by a parabolic distribution radially:

$$
V(R)=A\left(R-R_{t}\right)^{2}+V_{0}
$$

Here $V_{0}$ is the potential in the trough's minimum. The parameter $A$ can be estimated as follows: The potential wall height at about $1 \mathrm{~cm}$ distance from the trough minimum is about 5 Volts positive with respect to the minimum (see, for example, Figure 2 of ref. ${ }^{14}$, or Figure. 8 of the present work), which gives $A \approx 5 \mathrm{~V} / \mathrm{cm}^{2}$. The radial electric field is

$$
\partial V / \partial R=2 A\left(R-R_{t}\right)
$$


which gives $10^{3} \mathrm{~V} / \mathrm{m}$ for $R_{t}=15 \mathrm{~cm}$ and $R=16 \mathrm{~cm}$ (the curved path is $1 \mathrm{~cm}$ from trough minimum). With these numbers, and $Q=2$ for copper $^{37}$, the right-hand side of (7) amounts to $150 \mathrm{eV}$, even exceeding the left-hand side. This means that a trough height of 5 Volts is more than sufficient to generate a radial electric force opposing the centrifugal force acting on the ion.

The above example should serve the purpose to illustrate the effectiveness of a potential trough whose depth is much less than the volt-equivalent of the ion kinetic energy. It does not replace any of the plasma models developed in the literature because here formation and shape of the potential trough is not self-consistently dealt with and also the actual processes of plasma flow, involving collisions and electron-ion coupling, are not considered. For example, this picture also does not address the forces on electrons which increasingly drift to the filter coil if the coil has a higher positive bias than the optimum. Electron losses make the potential trough shallower, thereby reducing confinement and guiding of ions.

Concluding this topic, we point out that our simplified picture can readily explain one somewhat obscure feature, namely the reproducible, non-constant but "wavy" rate of plasma loss in a filter, as already observed by Storer et $\mathrm{al}^{29}$. When the transition from straight motion (before entering the filter) to approximately circular motion (inside the filter) occurs, ions will "overshoot" the equilibrium point of the average circular trajectory, and one can expect a flow oscillating around the equilibrium path; equilibrium understood as the location where the centrifugal force and the force caused by the radial electric field are balanced.

\section{SUMMARY AND CONCLUSIONS}

The overall efficiency of a $90^{\circ}$ - curved, open-coil filtered arc system has been assessed using the system coefficient $\kappa$ for pulsed and DC magnetic mode. Confirming previous findings, positive bias of the filter increases the available filtered ion current. The coefficient $\kappa$ saturates at about $15 \mathrm{~V}$ for relatively low magnetic field ( $\sim 10 \mathrm{mT})$. Stronger magnetic fields allow the coefficient $\kappa$ to be significantly higher; saturation of $\kappa$ occurs at higher bias of about $25 \mathrm{~V}$ for B $\sim 30-40 \mathrm{mT}$. These findings generally apply to both pulsed and DC filters. It has been shown that bias of pulsed filters can been realized simply by using the voltage drop across a self-bias resistor, which was electrically placed between the anode of the arc plasma source and the filter coil. This approach eliminated the need for a separate bias circuit and avoided the undesirable coupling of bias capacitor and pulse-forming-network, if the latter is used to drive the pulsed discharge. Under optimum conditions, almost $100 \mathrm{~A}$ of filtered copper ions have been obtained in pulsed mode, corresponding to $\kappa \approx 0.04$. No transport limit has been found when the arc current was increased.

Measurements with a floating probe indicated that the electric potential within a filter is characterized by a channel, or trough, which has a central dip of about 5-10 V and an additional potential "wall" near the filter coil. This potential distribution is a result of electron magnetization and lack of magnetization of ions; biasing determines the boundary conditions for the potential distribution. Ion transport can be accomplished even when the potential trough's depth is smaller than the volt-equivalent kinetic energy of ions because the radial electric field is strong enough to compensate the average centrifugal force acting on ions drifting through the curved filter.

\section{ACKNOWLEDGEMENTS}

The authors would like to thank Robert MacGill and Joe Wallig for technical assistance. This work was supported by the Assistant Secretary for Energy Efficiency and Renewable Energy, Office of Building Technology, of the U.S. Department of Energy under Contract No. DE-AC03-76SF00098. E. Byon was supported by the post-doctoral fellowship program of the Korean Science and Engineering Foundation (KOSEF). 


\section{References}

${ }^{1}$ R. L. Boxman, D. M. Sanders, and P. J. Martin, Handbook of Vacuum Arc Science and Technology (Noyes Publications, Park Ridge, N.J., 1995).

2 J. E. Daalder, J. Phys. D: Appl. Phys. 9, 2379 (1976).

3 B. Jüttner, IEEE Trans. Plasma Sci. PS-15, 474 (1987).

${ }^{4}$ B. Jüttner, J. Phys. D: Appl. Phys. 34, R103 (2001).

5 O. R. Monteiro, J. Vac. Sci. Technol. B 17, 1094 (1999).

${ }^{6}$ P. Siemroth, C. Wenzel, W. Kliomes, B. Schultrich, and T. Schülke, Thin Solid Films 308, 455 (1997).

7 P. J. Martin, R. P. Netterfield, T. J. Kinder, and L. Descotes, Surf. Coat. Technol. 49, 239 (1991).

8 A. Anders, Surf. Coat. Technol. 120-121, 319 (1999).

9 D. M. Sanders and A. Anders, Surf. Coat. Technol. 133-134, 78 (2000).

${ }^{10}$ R. L. Boxman, IEEE Trans. Plasma Sci. 29, $762-767$ (2001).

${ }^{11}$ P. J. Martin and A. Bendavid, Thin Solid Films 394, 1 (2001).

${ }^{12}$ I. I. Aksenov, V. A. Belous, V. G. Padalka, and V. M. Khoroshikh, Sov. J. Plasma Phys. 4, 425 (1978).

${ }^{13}$ I. I. Aksenov, V. A. Belous, and V. G. Padalka, Instrum. Exp. Tech. 21, 1416 (1978).

${ }^{14}$ M. M. M. Bilek, Y. Yin, and D. R. McKenzie, IEEE Trans. Plasma Sci. 24, 1165 (1996).

${ }^{15}$ I. I. Aksenov, S. I. Vakula, V. G. Padalka, V. E. Strel'nitskii, and V. M. Khoroshikh, Sov. Phys. Techn. Phys. 25, 1164 (1980).

${ }^{16}$ A. Anders, S. Anders, and I. G. Brown, J. Appl. Phys. 75, 4900 (1994).

${ }^{17}$ M. M. M. Bilek, D. R. McKenzie, Y. Yin, M. U. Chhowalla, and W. I. Milne, IEEE Trans. Plasma Sci. 24, 1291 (1996).

${ }^{18}$ T. Zhang, Y. C. Zhang, P. K. Chu, and I. G. Brown, Appl. Phys. Lett. 80, 365 (2002).

${ }^{19}$ V. N. Zhitomirsky, L. Kaplan, R. L. Boxman, and S. Goldsmith, Surf. Coat. Technol. 76, 190 (1995).

${ }^{20}$ A. Anders, S. Anders, and I. G. Brown, Plasma Sources Sci. Technol. 4, 1 (1995).

${ }^{21}$ M. M. M. Bilek, J. Appl. Phys. 85, 6385 (1999).

${ }^{22}$ X. Shi, B. G. Tay, and S. P. Lau, Int. J. Mod. Phys. B 14, 136 (2000).

${ }^{23}$ A. Anders and R. A. MacGill, Surf. Coat. Technol. 133-134, 96 (2000).

${ }^{24}$ B. Alterkop, E. Gidalevich, S. Goldsmith, and R. L. Boxman, J. Phys. D: Appl. Phys. 29, 3032-3038 (1996).

${ }^{25}$ B. Alterkop, E. Gidalevich, S. Goldsmith, and R. L. Boxman, J. Phys. D: Appl. Phys. 31, 873-879 (1998).

${ }^{26}$ G. F. You, B. K. Tay, S. P. Lau, D. H. C. Chua, and W. I. Milne, Surf. Coat. Technol. 150, 50-56 (2002).

${ }^{27}$ R. A. MacGill, M. R. Dickinson, A. Anders, O. R. Monteiro, and I. G. Brown, Rev. Sci. Instrum. 69, 801-803 (1998).

${ }^{28}$ A. Anders, R. A. MacGill, and T. A. McVeigh, Rev. Sci. Instrum. 70, 4532-4534 (1999).

${ }^{29}$ J. Storer, J. E. Galvin, and I. G. Brown, J. Appl. Phys. 66, 5245-5250 (1989).

${ }^{30}$ E. M. Oks, A. Anders, I. G. Brown, M. R. Dickinson, and R. A. MacGill, IEEE Trans. Plasma Sci. 24, 1174-1183 (1996).

${ }^{31}$ A. Anders, IEEE Trans. Plasma Sci. 30, 108-109 (2002).

${ }^{32}$ A. I. Morozov, Sov. Phys. Doklady 10, 775-777 (1966).

${ }^{33}$ D. B. Boercker, D. M. Sanders, J. Storer, and S. Falabella, J. Appl. Phys. 69, 115-120 (1991).

${ }^{34}$ A. A. Plyutto, V. N. Ryzhkov, and A. T. Kapin, Sov. Phys. JETP 20, 328-337 (1965).

${ }^{35}$ W. D. Davis and H. C. Miller, J. Appl. Phys. 40, 2212-2221 (1969).

${ }^{36}$ A. Anders and G. Y. Yushkov, J. Appl. Phys. 91, 4824-4832 (2002).

${ }^{37}$ I. G. Brown, Rev. Sci. Instrum. 65, 3061-3081 (1994). 